\title{
Large-Eddy Simulation of Turbulent Combustion
}

\author{
Heinz Pitsch \\ Department of Mechanical Engineering \\ Stanford University \\ Stanford, CA 94305-3030 \\ H.Pitsch@stanford.edu
}

In recent years, Large Eddy Simulation (LES) has been successfully applied to non-premixed and premixed turbulent combustion problems $[1,2,3]$. In most technical combustion applications, the pure non-premixed or premixed combustion models are no longer valid, since partially premixed combustion has to be taken into account. An example is the stabilization region of a lifted non-premixed flame. To overcome this problem, a Combined Conserved Scalar/Level-Set Flamelet model has been proposed [4, 5], which allows for the computation of reactive flows, where both premixed and non-premixed combustion occurs. In this formulation, the $G$-equation method is used to describe partially premixed flame propagation and to distinguish between burned and unburned regions. The reacting gases in the post-flame region are described by a laminar diffusion flamelet approach. The principal roadmap for the further development and validation of these models is to develop and validate the appropriate methods for the pure premixed and non-premixed combustion situations, and combine the resulting models for a more general formulation. Much of this work has been done in the past. We have developed unsteady flamelet models for LES of non-premixed turbulent combustion [1,2], and a level-set method for LES of premixed turbulent combustion [3]. These models have been validated with premixed and non-premixed turbulent jet flame experiments. Preliminary simulations with a Combined Conserved Scalar/Level-Set Flamelet Model have already been performed [6, 5]. Results from these simulations are shown in Figs. 1 and 2. Recently, we have developed a new consistent formu-
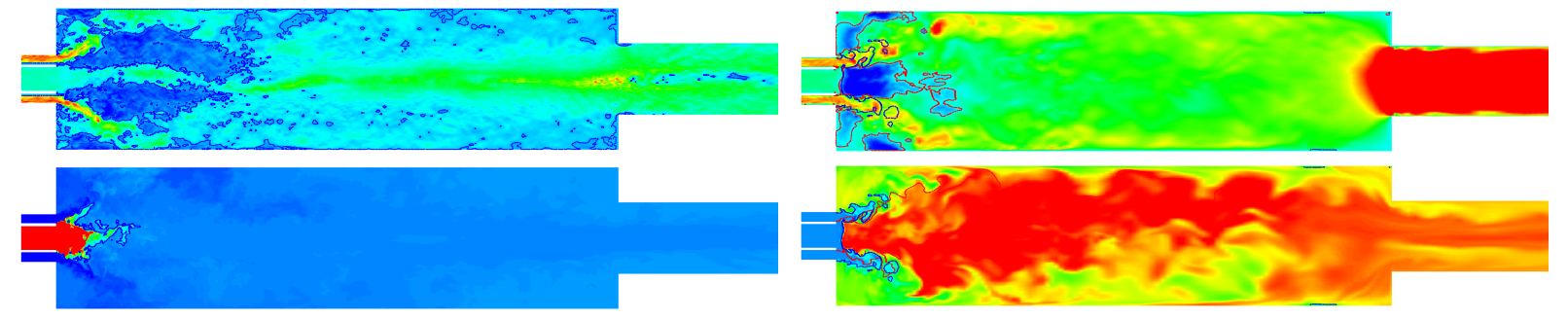

Figure 1: Left: Axial velocity (top) and mixture fraction (bottom) from the cold flow simulation. The blue lines are zero axial velocity and stoichiometric mixture fraction in the velocity and the mixture fraction figure, respectively. Right: Axial velocity (top) and temperature (bottom) from the fired case simulation. Blue lines represent the instantaneous flame surface, red lines are zero axial velocity and stoichiometric mixture fraction in the velocity and the temperature figure, respectively
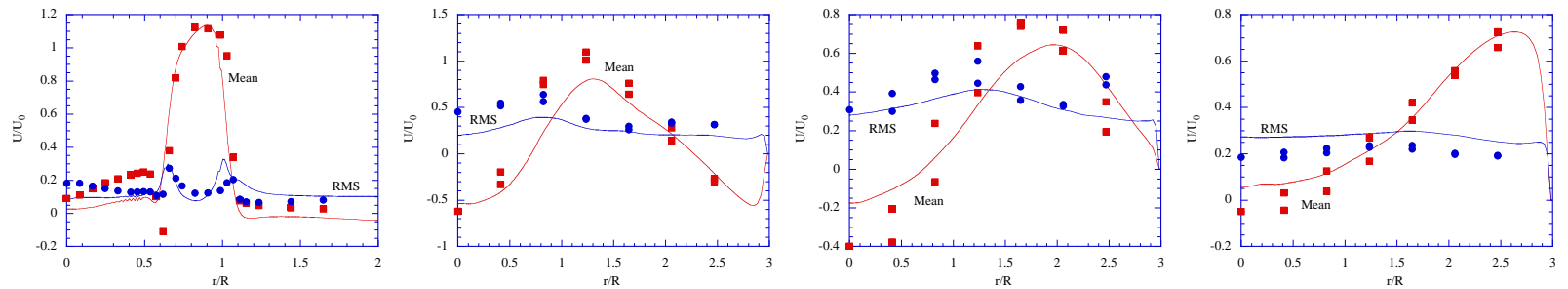

Figure 2: Axial velocities from the fired simulation (lines) compared with experimental data (symbols) at $x / R=0.08, x / R=1.8, x / R=3.3$, and $x / R=6.3$ from left to right

lation of the $G$-equation approach for LES [7]. The solution of the instantaneous unfiltered $G$-equation has physical meaning only at the instantaneous flame front location. The definition of the remaining $G$-field is arbitrary. Hence, in a filtering procedure, applied to derive the appropriate LES equation, only states on the 
instantaneous unfiltered flame surface can be considered, which excludes the use of conventional volumetric LES filters. A new filter kernel has been developed, which averages along surfaces. Using this filter, the $G$-equation for the filtered flame front location can be derived This equation has two unclosed terms. Since the new filter kernel only considers states at the instantaneous flame front, the convection velocity appearing in this equation is an average conditioned on the location of the flame surface. To relate this conditional velocity to the unconditionally Favre-filtered velocity $\widetilde{\boldsymbol{v}}$, which is known from the solution of the momentum equations, a model for this quantity has also been developed. The resulting equation for the filtered flame front location is

$$
\frac{\partial \check{G}}{\partial t}+\widetilde{\boldsymbol{v}} \cdot \nabla \check{G}=\frac{\rho_{u}}{\bar{\rho}} s_{T, u}|\nabla \check{G}| .
$$

The second unclosed term involves the turbulent burning velocity. A model for this term has also been developed. A length scale equation for the sub-grid flame brush thickness has been derived. An analytic expression for the turbulent burning velocity follows from the assumption that production equals dissipation in this equation. Interestingly, because of the use of the new filtering procedure, a propagation term proportional to the curvature of the mean front, which appeared in earlier formulations of the filtered $G$-equation, is no longer present in Eq. (1). This is an important difference, since the curvature term has a stabilizing effect on the flame front and therefore leads to a decreased resolved flame wrinkling. The model is validated by simulations of three turbulent premixed Bunsen flames at different Reynold numbers. Earlier simulations for the lower Reynolds number case using a different level set formulation resulted in an underprediction of the flame brush thickness. The results of the present simulations are compared with the earlier simulations and experimental data for temperature, velocity field, and turbulent flame brush thickness.

\section{References}

[1] H. Pitsch and H. Steiner. Large-eddy simulation of a turbulent piloted methane/air diffusion flame (Sandia flame D). Phys. Fluids, 12(10):2541-2554, 2000.

[2] H. Pitsch. Improved pollutant predictions in large-eddy simulations of turbulent non-premixed combustion by considering scalar dissipation rate fluctuations. Proc. Combust. Inst., 29:1971-1978, 2002.

[3] H. Pitsch and L. Duchamp de Lageneste. Large-eddy simulation of premixed turbulent combustion using a level-set approach. Proc. Combust. Inst., 29:2001-2008, 2002.

[4] N. Peters. Turbulent Combustion. Cambridge University Press, 2000.

[5] H. Pitsch, P. Trouillet, C. D. Pierce, E. Tribbett, C. M. Sipperley, C. F. Edwards, and C. T. Bowman. A joint experimental/large-eddy simulation study of a model gas turbine combustor. Western States Section Meeting of the Combustion Institute, 2002, San Diego, CA, pages WSSCI 02S-59, 2002.

[6] H. Pitsch and L. Duchamp de Lageneste. Large-eddy simulation of lifted turbulent diffusion flames. $2^{\text {nd }}$ Joint meeting of the US sections of the Combustion Institute, Oakland, CA, page paper 263, 2001.

[7] H. Pitsch and L. Duchamp de Lageneste. A g-equation formulation for large-eddy simulations of premixed turbulent combustion. CTR Annual Research Briefs, pages 3-14, 2002. 